\title{
Protoporphyrinogen Oxidase Inhibitor: An Ideal Target for Herbicide Discovery
}

\author{
Ge-Fei Hao, Yang Zuo, Sheng-Gang Yang, and Guang-Fu Yang*
}

\begin{abstract}
As the last common enzyme in the biosynthetic pathway leading to heme and chlorophyll, protoporphyrinogen oxidase (PPO; EC 1.3.3.4) is an ideal target for herbicide development. Currently, about 30 PPO inhibitors have been developed as agricultural herbicides. PPO inhibitors have displayed environmentally benign, but advantageous characteristics, including low toxicity, low effective concentration, broad herbicidal spectrum (active against both monocotyledon and dicotyledon weeds), quick onset of action, and long lasting effect. Over the last several years, great achievements have been made in revealing the structural biology of PPO. Five PPO crystal structures, four isolated in enzyme-inhibitor complexes and one in the native form, have been determined, including those from Nicotiana tabacum, Myxococcus Xanthus, Bacillus subtilis, and human. Although PPO inhibitors have been developed for over forty years, we continue to uncover exciting future prospects for novel PPO-inhibiting herbicides. In this review, we have summarized the structures of PPOs from plants, human, and bacteria; the interactions between PPOs and inhibitors; the quantitative structure-activity relationships of PPO inhibitors; and the molecular design of new PPO inhibitors.
\end{abstract}

Keywords: Herbicide $\cdot$ Inhibitor · Molecular design · Protoporphyrinogen oxidase $\cdot$ QSAR

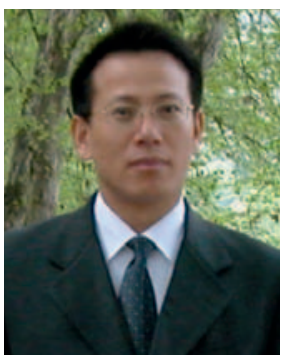

Guang-Fu Yang (born in 1970) got his Bachelor degree of Science in Chemistry at the Department of Chemistry, Central China Normal University in 1992 and his Doctor of Philosophy in Organic Chemistry from Institute of ElementOrganic Chemistry, Nankai University in 1997. He was a visiting Professor at College of Pharmacy, University of Kentucky from August 2004 to February 2005. Since July 1997, he has been at the faculty of Central China Normal University and promoted as

${ }^{*}$ Correspondence: Prof. G.-F. Yang

College of Chemistry, Central China Normal University Key Laboratory of Pesticide \& Chemical Biology of

Ministry of Education

Wuhan 430079, P. R. China

Tel.: +862767867800

Fax: +86 2767867141

E-mail: gfyang@mail.ccnu.edu.cn associate Professor in 1998 and Professor in 2001. Since 2002, he was selected as the Dean of College of Chemistry, Central China Normal University and the director of Key Laboratory of Pesticide \& Chemical Biology of Ministry of Education. In 2009, he was awarded the National foundation for distinguish young scientist from NSFC and the award of National Model Teacher. His research interests includes rational design of green pesticide and its chemical biology, quantitative structure-activity relationships of bioactive compounds, and heterocyclic chemistry.

\section{Introduction}

Protoporphyrinogen oxidase (PPO; EC 1.3.3.4) is the last common enzyme in the biosynthetic pathway leading to heme and chlorophyll. ${ }^{[1-3]}$ In the presence of molecular oxygen, PPO catalyzes the sixelectron oxidation of protoporphyrinogenIX to the fully conjugated macrocyclic protoporphyrin IX (Scheme 1). PPO has been found in mammals, plants, fungi, and bacteria. It represents a highly conserved family of membrane-associated enzymes. In plants, there are two isoforms of PPO; the plastidic PPO1 and the mitochondrial PPO2. ${ }^{4]}$ PPO1 is located in the thylakoid and in the envelope membranes of chloroplasts; PPO2 is situated on the outer surface of the inner mitochondrial membrane.

In humans, defects in the $P P O$ gene cause a dominantly inherited metabolic disease called variegated porphyria (VP). VP symptoms include acute abdominal pain, neurological manifestations, and/ or cutaneous photosensitivity. ${ }^{[5-9]} \mathrm{VP}$ is more common in women than in men, and it may suddenly occur at any age, from adolescence to old age.[10] It has been reported that PPO activity is reduced by at least $50 \%$ in patients with VP. ${ }^{[11]}$ Humans become more sensitive to light when the activity of PPO is reduced by mutations. The subsequent protoporphyrin

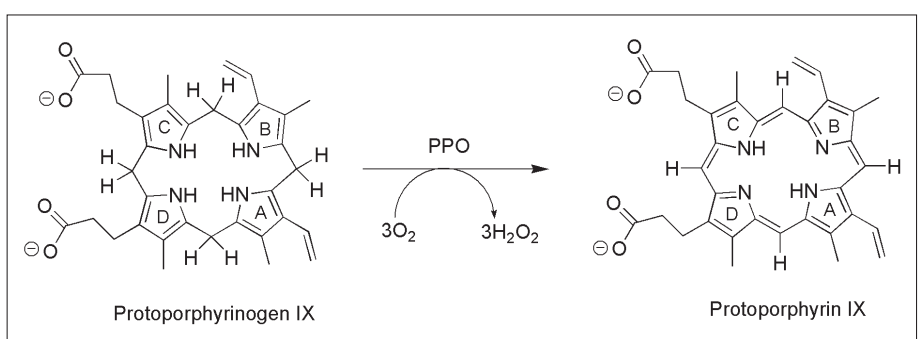

Scheme 1. Protoporphyrinogen oxidase (PPO) catalyzes the oxidation reaction of protoporphyrinogen IX (left) with molecular oxygen to produce protoporphyrin IX (right). In this reaction, the molecular oxygen is reduced to hydrogen peroxide, and six hydrogen atoms are eliminated. 
IX accumulation causes VP. Genetic studies of patients with VP have shown that one amino acid mutation (e.g. R59W) near the active site of the PPO enzyme could cause the disease. Interest in PPO increased substantially with the finding that PPO inhibitors showed potential in the treatment of cancer via photodynamic therapy (PDT). ${ }^{[12]}$ Halling et al. ${ }^{[13]}$ showed that PPO inhibitors could activate the photosensitive protoporphyrinogen-IX and cause it to accumulate within tumor cells.

In plants, PPO was identified as a significant target for structurally diverse herbicides, including diphenylethers, ${ }^{[3]}$ phenylpyrazoles, ${ }^{[14]}$ oxadiazoles, ${ }^{[15]}$ triazolinones, ${ }^{[16]}$ thiadiazoles, ${ }^{[17]}$ pyrimidindiones, ${ }^{[18]}$ oxazolidinedione, ${ }^{[19]} \mathrm{N}$-phenylphthalimides, ${ }^{[20]}$ and others. ${ }^{[21]}$ Inhibition of the plant enzyme caused accumulation of the protoporphyrinogen-IX substrate, which was exported to the cytoplasm, where it was nonenzymatically oxidized by $\mathrm{O}_{2}$ in the mitochondrion and chloroplast. This produced a photosensitive protoporphyrin IX. With exposure to light, this protoporphyrin IX generated singlet oxygen molecules that caused lipid peroxidation and cell death. Therefore, PPO-inhibiting herbicides are also known as light-dependent bleaching herbicides. ${ }^{[22]}$

In this review, we will summarize current knowledge on the structure of PPOs from plants, humans, and bacteria; the interactions between PPOs and inhibitors; the quantitative structureactivity relationships (QSARs) of PPO inhibitors; and the molecular design of new PPO inhibitors.

\section{Structure of PPOs}

The three-dimensional structure of an enzyme provides the basis for understanding the enzymatic reaction mechanism, the inhibitor-enzyme interaction mechanism, and the design of new inhibitors. In 2004, Koch et al. ${ }^{[14]}$ described the first crystal structure of mitochondrial PPO2 from common tobacco (Nicotiana tabacum) (mtPPO) in complex with the inhibitor, 4-bromo-3-(5'-carboxy-4'-chloro-2'-fluorophenyl)-1-methyl-5-trifluoromethyl-pyrazol (INH, see fluazolate in Fig. 1). The structure was observed at $2.9 \AA$ resolution with selenium single anomalous diffraction. This dimeric yellow protein consisted of three domains; a FAD-binding domain, with a topology like that of $p$-hydroxybenzoate hydroxylase; a substratebinding domain, which enclosed a narrow, active site cavity beneath the FAD; and an $\alpha$-helical, membrane-binding domain. Subsequently, the crystal structures were successfully determined for the native PPO from bacteria (Myxococcus xanthus;
mxPPO), in complex with the inhibitor acifluorfen (AF, Fig. 1); ${ }^{[23]}$ the PPO from Bacillus subtilis (bsPPO), in complex with $\mathrm{AF} ;{ }^{[24]}$ and the human PPO (hPPO), also bound to $\mathrm{AF}^{[25]}$ The mtPPO, mxPPO, and hPPO are very similar, membrane-bound dimers, and they are strongly inhibited by typical diphenylether herbicides, like AF. However, bsPPO is very unique in the PPO protein family; it is monomeric and cytoplasmic, it has much broader substrate specificity, and it is not inhibited by AF.

The sequence alignment showed that the sequence identity is very low among mPPO, mxPPO, bsPPO, and hPPO. However, they have very similar overall folding patterns, as defined by Koch et al. ${ }^{[14]}$ They all possess an FAD-binding domain, a substrate-binding domain, and membrane-binding domain. The structure of the FAD-binding domain showed significant amino acid sequence and structural homology to other flavoenzymes. ${ }^{[14]}$ When the substrate-binding domain of mtPPO was superimposed onto mxPPO, bsPPO, and hPPO, the root-mean-square deviations (RMSD) were $0.7 \AA, 1.1 \AA$, and $0.8 \AA$ for the $\mathrm{C} \alpha$ atoms, respectively. In addition, among the four PPO enzymes, the conformation of the FAD-binding and substrate-binding domains were nearly identical, except that a few loop regions were different, most likely altered by amino acid insertions and deletions. ${ }^{[14,23-25]}$ In contrast, the conformation of the membrane-binding domain was significantly different among the four PPO enzymes; this was presumably related to their different modes of interacting with the membrane. For example, based on the crystal forms, mtPPO was proposed to interact with the membrane in the dimer conformation, ${ }^{[14]}$ but mxPPO did not form a physiologically relevant dimer. It was also proposed that the hydrophobic interface between helices 4, 5, and 10 in the membrane-binding domains of mxPPO formed a membrane anchor. ${ }^{[23]}$

The active site of PPO is a hydrophobic cavity located at the interface between the FAD-binding and substrate-binding domains; this site has some well-conserved, functional, amino acid residues. ${ }^{[14,23-25]}$ As shown in Fig. 2, the most highly conserved residue is the glycine at position 175 in mtPPO, 167 in mxPPO, 169 in hPPO,

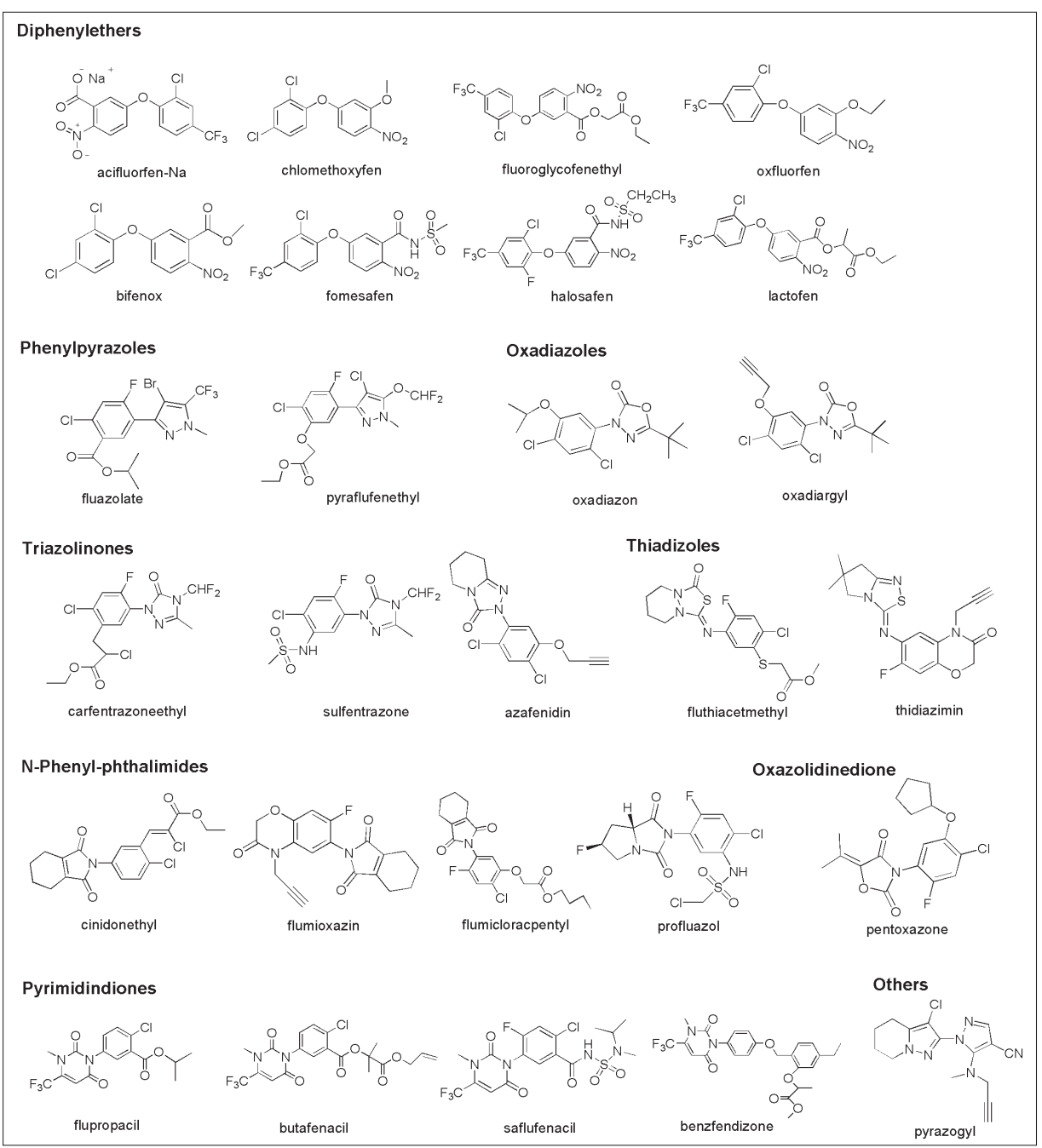

Fig. 1. Chemical structures of representative protoporphyrinogen oxidase-inhibiting herbicides (www.weedscience.org). 


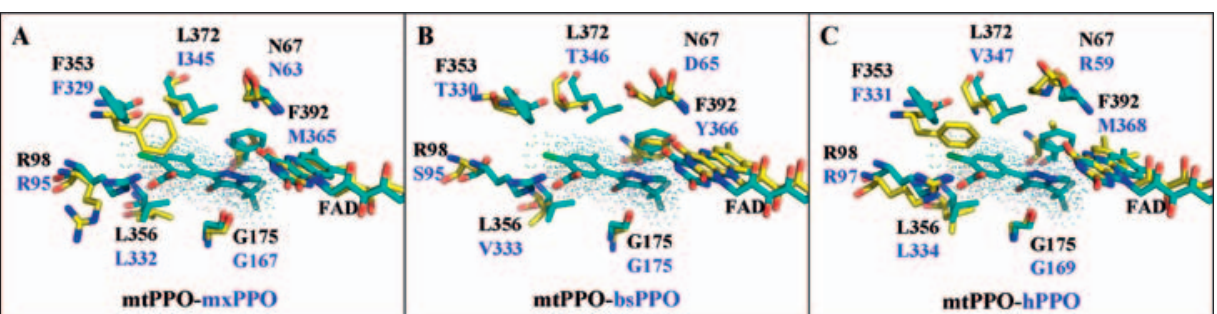

Fig. 2. Superimposition of the active sites of PPOs. (A) Mitochondrial and bacterial PPOs (mtPPOmxPPO); (B) mitochondrial and bacterial PPOs (mtPPO-bsPPO); (C) mitochondrial and human PPOs (mtPPO-hPPO).

and 175 in bsPPO. Its carbonyl oxygen protrudes from the floor of the cavity to the center of the active site, and it interacts with the tetrapyrrole macrocyclic structure. Another highly conserved residue is the arginine at position 98 in mtPPO, 95 in mxPPO, and 97 in hPPO. The equivalent residue in bsPPO is not arginine, but Ser95. According to the structural modeling of the PPO bound to a substrate, this residue was proposed to interact ionically with the propionic acid group of the $\mathrm{C}$ ring; it may form an H-bond with the carboxyl group of the propionic acid. The conservation of this bulky residue on one side of the pocket has presumably constrained the size of the residue on the other side, because that residue is always a relatively small, uncharged residue, like Ala, Gly, Thr, or Ser. The third most highly conserved residue is Phe353, which is equivalent to Phe329 in mxPPO, Phe331 in hPPO, and Thr330 in bsPPO. It is optimally placed to limit the height of the ceiling of the cavity on the opposite side of the macrocyclic structure of FAD.

Fig. 2 also shows the other important residues in the active site. These are not highly conserved, but they show similarity in type or size among the different PPO species. For example, Phe392 in mtPPO is stacked with ring A of protoporphyrinogen-IX. ${ }^{[14]}$ This residue is replaced by Met365 in mxPPO and Tyr366 in bsPPO. Interestingly, the Asn67 in mtPPO, positioned at the top of the isoalloxazine ring of FAD, is replaced by Asn63 in mxPPO, Asp65 in bsPPO, and Arg59 in hPPO. The Arg59 in hPPO has attracted a lot of attention, due to its essential role in maintaining enzymatic activity and structural stability in vitro. Before the crystal structure of hPPO had been solved, it was proposed, based on the mIPPOX structure, that the Arg59 in hPPO formed a salt bridge with Asp349, which contributed to the integrity of the active site. ${ }^{[14]}$ Among various hPPO mutants, the R59W mutation is most commonly studied, due to its significant association with VP. Based on the crystal structure of hPPO, Qin et al.[25] showed that the hydrophilic binding site repelled the indolyl ring of tryptophan, and this greatly weakened the interaction between the isoalloxazine ring of FAD and the substrate. In addition, the binding site harbors a pair of non-conserved residues; these are Leu356 and Leu372 in mtPPO, Leu332 and Ile345 in mxPPO, Val333 and Thr346 in bsPPO, and Leu334 and Val347 in hP$\mathrm{PO}$. The $\mathrm{B}$ ring of protoporphyrinogen-IX is sandwiched between these two residues and forms hydrophobic interactions.

Interestingly, PPO enzymes from different species exhibit a variety of substrate specificities due to the different sizes of substrate binding cavities. Qin $e t$ al. ${ }^{[24]}$ demonstrated that bsPPO, mtPPO, and mxPPO had volumes of $1,173 \AA^{3}, 440$ $\AA^{3}$, and $627 \AA^{3}$, respectively. The bsPPO binding cavity can accommodate 2 - to 3-fold larger substrates than mtPPO and mxPPO. They also found that positive charges lined the surface of the bsPPO substrate binding chamber, but not the surfaces of the mtPPO and mxPPO binding chambers. These findings could explain why bsPPO has broader substrate specificity than other PPO enzymes.

To date, the true binding mode of protoporphyrinogen-IX remains unclear, because no one has solved the crystal structure of PPO in complex with protoporphyrinogen-IX or its analogues. Based on the crystal structure of mtPPO in complex with the inhibitor INH, Koch et al. ${ }^{[14]}$ proposed the first detailed binding mode of protoporphyrinogen-IX. They showed that two negatively charged propionyl groups were oriented towards the solvent-exposed parts of the cavity, and the propionyl groups of the $\mathrm{C}$ rings interacted ionically with the highly conserved residue, Arg98. The A ring formed an aromatic stack with Phe392, and the B ring was sandwiched be(hPPO); (C) AF in bacterial PPO (bsPPO). tween the two conserved residues, Leu356 and Leu372. This binding mode orients the methylene bridge (C20) between rings $\mathrm{A}$ and D towards the reactive N5 atom of the FAD; this led to the assumption of a threestep oxidation mechanism. ${ }^{[14]}$ The key of the proposed mechanism was that hydride abstraction reactions always occurred at the $\mathrm{C} 20$ atom of the tetrapyrrol ring by hydrogen rearrangements through imineenamine tautomerizations. Although this mechanism has been widely accepted, some important questions remain unclear. For example, protoporphyrinogen IX can be nonenzymatically oxidized by $\mathrm{O}_{2}$, but the rate-determining step of this nonenzymatic transformation remains unknown. It is not clear what the difference is between the enzymatic and nonenzymatic reaction. Moreover, it is unknown whether the ratedetermining step involves substrate binding or leaving.

It is known that existing inhibitors compete with the substrate by mimicking parts of the substrate structure. Interestingly, different inhibitors have different mimicking modes, and the same inhibitor might have different binding modes with PPOs from different species. For example (Fig. 3), INH mimics rings $\mathrm{A}$ and $\mathrm{B}$ of protoporphyrinogen-IX, and the INH carboxylate group stands in for the propionate group of ring C.[14] The crystal structures revealed that AF could mimic rings $\mathrm{A}$ and $\mathrm{B}$ of the substrate in mxPPO ${ }^{[23]}$ and $\mathrm{hPPO},{ }^{[25]}$ but it mimicked rings $\mathrm{C}$ and $\mathrm{D}$ of the substrate in bsPPO. [24] These results indicated that two AF molecules could mimic all four rings of protoporphyrinogen-IX. This has provided new insights for future inhibitor design.

\section{PPO Inhibitors as Herbicides}

PPO-inhibiting herbicides have been extensively investigated for over 40 years. However, until the mid-1980s, the mode of action for this family of herbicides was unknown. In contrast to other herbicides, PPO inhibitors have many advantages, including low toxicity, a low effective concentration (10 50 g.ai/ha), a broad herbicidal spectrum (active against both

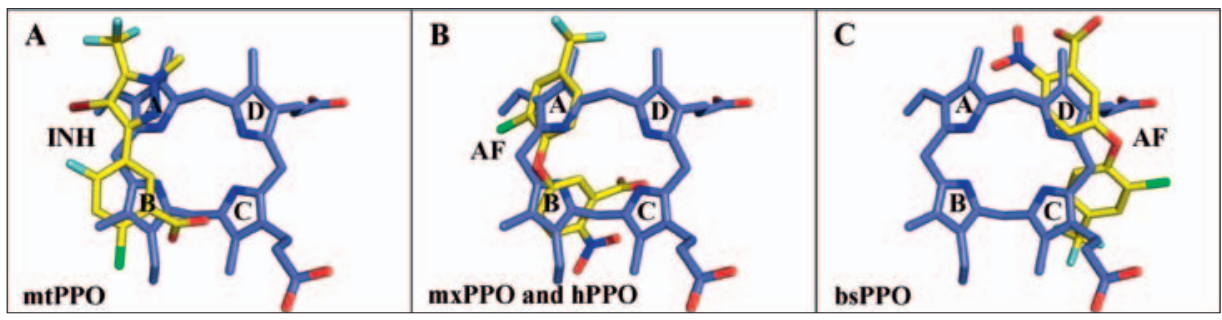

Fig. 3. Inhibitor modes can mimic different protoporphyrinogen oxidases (PPOs). (A) INH in mitochondrial PPO (mtPPO); (B) Acifluorfen (AF) in PPOs from bacteria (mxPPO) and human 
monocotyledon and dicotyldon weeds), a quick onset of action (necrosis within $24 \mathrm{~h}$ ), a long lasting effect, and other environmentally benign characteristics. During the last few decades, several chemical families, including diphenylethers, ${ }^{[3]}$ phenylpyrazoles, ${ }^{[14]}$ oxadiazoles, ${ }^{[15]}$ triazolinones, ${ }^{[16]}$ thiadiazoles, ${ }^{[17]}$ pyrimidindiones, ${ }^{[18]}$ oxazolidinedione, ${ }^{[19]}$ and $N$-phenyl-phthalimides, ${ }^{[20]}$ have been developed as commercial herbicides that targeted PPOs. The chemical structures of some representative commercial PPO-inhibiting herbicides are listed in Fig. 1.[26]

Compared to other herbicides, PPOinhibiting herbicides cause resistance to develop at a much slower rate. Although this family of herbicides was first commercialized in the 1960s, only four weeds have been reported that developed resistance to PPO-inhibiting herbicides. ${ }^{[27]}$ Amaranthus tuberculatus, one of the most problematic dicot weeds in agronomic fields throughout the midwestern United States, was first reported in 2001 to have developed a cross-resistance to PPO and acetohydroxyacid synthase (AHAS, EC 4.1.3.18) inhibitors. Subsequently, three other PPO inhibitor-resistant dicot weeds were identified, Euphorbia heterophylla, A. quitensis, and Ambrosia artemisiifolia. Interestingly, the development of resistance to PPO-inhibiting herbicides was, in part, attributable to the development of a resistance to AHASinhibiting herbicides, ${ }^{[28]}$ because farmers used PPO-inhibiting herbicides to control weeds resistant to AHAS-inhibiting herbicides. For example, A. tuberculatus is known to be resistant to PPO inhibitors, acifluorfen-Na, fomesafen, lactofen, and the AHAS inhibitors, chlorimuron-ethyl, imazethapyr, and thifensulfuron-methyl. Recently, Patzoldt et al. ${ }^{[29]}$ showed that, within natural populations of $A$. tuberculatus, the selected mechanism of PPO inhibitor resistance was the Gly210 codon deletion of the PPX2L gene, which was predicted to encode both plastid- and mitochondria-targeted PPO isoforms. This unique mechanism, an amino acid deletion, rather than a substitution, was of great interest, because the most common mechanism for conferring resistance is an alteration in the target site. An integration of homology modeling, molecular dynamics (MD) simulations, and molecular mechanics-Poisson-Boltzmann surface area (MM-PBSA) calculations provided a plausible description of the detailed molecular mechanism of drug resistance associated with Gly210. ${ }^{[30]}$ The results indicated that, although Gly210 in the wild-type A. tuberculatus PPO has no direct interaction with the inhibitors, a Gly210 deletion had a great effect on the hydrogen-bonding network and it changed the conformation of the binding pocket. In the wild-type A. tuberculatus PPO, Gly 210 formed an inter-chain hydrogen bond with Ser424, which played an important role in stabilizing the local conformation of the wild-type enzyme. As shown in Fig. 4, after the Gly210 deletion, this important hydrogen bond disappeared, which in turn caused a change in the conformation of the binding pocket. This change significantly weakened the favorable hydrogen bonding interactions between residue Arg128 and the inhibitors. As a result, the mutated PPO had reduced affinity for the inhibitor, which conferred herbicide resistance.

Recently, the PPO inhibitor-resistant crop has emerged as a hot area of investigation. ${ }^{[31,32]}$ Emphasis has been placed on conventional tissue culture methods, the expression of modified co-factors for the protoporphyrin IX binding subunit proteins, and overexpression of the wild-type plant PPO gene. Some PPO inhibitor-resistant crops have been reported, including tobacco, soybean, tomato, rice, and maize. ${ }^{[31]}$ The resistance level has ranged from 2-fold to 1000 -fold. Due to the highly diverse chemical structures of PPO inhibitors, the development of PPO-inhibitor resistance technology has not depended on any single herbicide or mutant PPO gene. For example, some attempts have been made to develop PPO herbicide-removal systems, including P-450 monooxygenases ${ }^{[33]}$ and chelatase approaches. ${ }^{[34]}$ The P-450 monooxygenase approach has been explored in the development of herbicideresistant crops, but a major question arose when it was applied to PPO herbicides, because the PPO-inhibiting herbicide is rapid-acting. Thus, a detoxification strategy might be too slow to confer resistance to rapid-acting herbicides. Instead, a P-450 monooxygenase should be developed with highly efficient catalytic activity that can also adapt to the chemical diversity of PPO inhibitors.

\section{QSAR of PPO Inhibitors}

Since around 1970, when oxadiazon and chlorophthalim were patented, numerous compounds have been synthesized and evaluated for their herbicidal and PPO inhibition activities. Several QSAR calculations have been formulated to reveal the interaction mechanism of PPO inhibitors and guide structural optimization. A comprehensive review by Fujita et al. [35] summarized the progress of QSAR studies with PPO inhibitors, including diphenylethers, thiadiazoles, $\quad N$-phenylphthalimides, $\mathrm{N}$-phenyltriazolinones, $\mathrm{N}$-phenyltriazolinethiones, and $N$-phenyltetrahydroindazoles.

It should be pointed out that the quality of a QSAR equation is highly dependent upon the choice of descriptors. For example, $N$-phenyltriazolinones are one of the most important PPO inhibitors discovered by scientists at the FMC Corporation. This series includes sulfentrazone, a pre-emergency soybean herbicide, and carfentrazone-ethyl, a post-emergency cereal herbicide. Theodoridis et al. ${ }^{[36]}$ of the FMC Corporation performed a QSAR

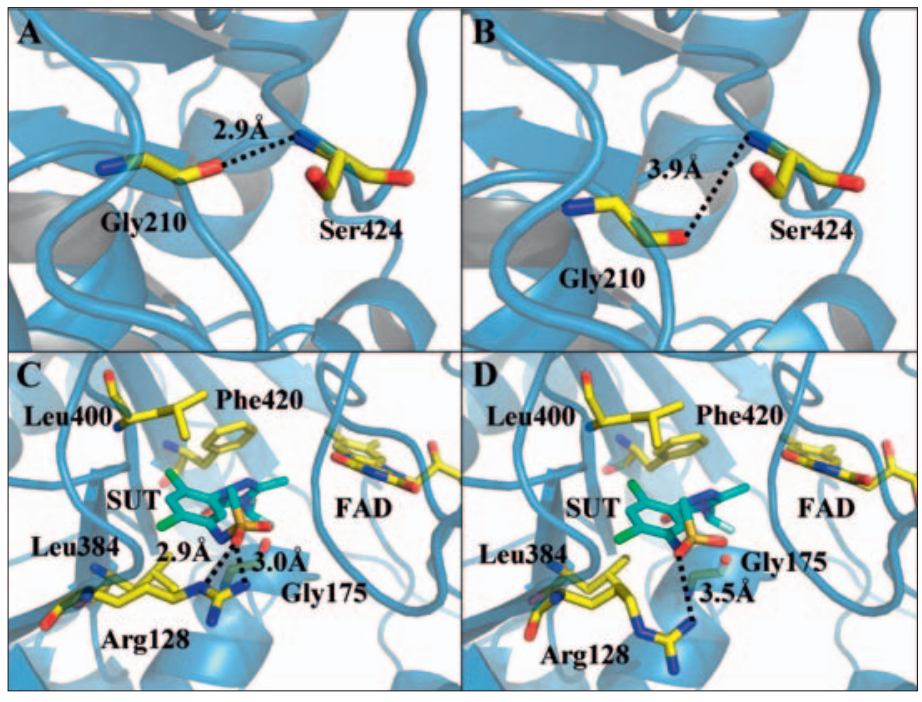

Fig. 4. Comparison of hydrogen bonding interactions (dotted lines) between the mutant and wild-type $A$. tuberculatus protoporphyrinogen oxidases (PPOs). (A) The interchain hydrogen bond between Gly210 and Ser424 in wild-type PPO. (B) The distance between Gly210 (corresponding to Gly211 in wild-type enzyme) and Ser424 in mutant PPO. (C) In the wild-type complex, two hydrogen bonds formed between the inhibitor, sulfentrazone (SUT, light blue), and Arg128. (D) In the mutant complex, no hydrogen bond formed between the inhibitor, sulfentrazone, and Arg128. 
analysis for hydroponically measured herbicidal activity against cucumber seeding for a series of 1-substitutedphenyl-4H-1,2,4-triazolin-5-ones (Table 1). Two physicochemical descriptors, the hydrophobicity term, $\pi$, and the STERMOL term, $B_{1}$, were examined for correlations with the biological activity, $p \mathrm{I}_{50}$ (-log concentration in mol/liter). Fourteen herbicides with substituents at position $11\left(\mathrm{R}^{2}\right)$ of the phenyl ring (1-14 in Table 1) were evaluated with Eqn. (1) as follows:

In this and the following QSAR equations, $\mathrm{n}$ is the number of compounds included in the analysis, $r^{2}$ is the correlation coefficient, $s$ is the standard error, $F$ is the F-test value, and the figures in parentheses are the $95 \%$ confidence intervals of the regression coefficient. However, when the predictability of Eqn. (1) was examined for five additional compounds (15-19 in Table 1), this equation only predicted the herbicidal activity of three compounds $\left(\mathbf{1 5}, \mathbf{1 6}\right.$, and 18). Therefore, Fujita et al. ${ }^{[35]}$

$$
\begin{aligned}
& p \mathrm{I}_{50}=2.51+7.02 B_{1}( \pm 2.351)-2.57( \pm 0.775) B_{1}^{2}-0.18( \pm 0.045) \pi^{2} \\
& \mathrm{n}=14, r^{2}=0.78 \text {, and } s=0.216
\end{aligned}
$$

$$
\begin{aligned}
& p \mathrm{I}_{50}=7.135( \pm 0.299)+0.211( \pm 0.123) \Delta L-0.780( \pm 0.334) \Delta B_{1}-0.123( \pm 0.066)-B_{5} \\
& \mathrm{n}=18, r^{2}=0.67, s=0.240, F=9.46
\end{aligned}
$$

$$
\begin{aligned}
& p \mathrm{I}_{50}=4.1272( \pm 0.0673)+2.6437( \pm 0.3409) Q_{C_{11}}+0.0520( \pm 0.0125) \\
& +0.0495( \pm 0.0096) \\
& \mathrm{n}=24, r^{2}=0.86, s=0.330, F=40.67
\end{aligned}
$$

reexamined the QSAR of this series of compounds and formulated Eqn. (2) for 18 compounds (1-18).

\begin{tabular}{|c|c|c|c|c|c|c|c|c|c|}
\hline \multirow[t]{2}{*}{ No. } & \multirow[t]{2}{*}{$\mathrm{R}^{1}$} & \multirow[t]{2}{*}{$\mathrm{R}^{2}$} & \multicolumn{7}{|c|}{$\mathrm{pI}_{50}$} \\
\hline & & & Obs & $\begin{array}{l}\text { Eqn. } \\
\text { (1) }\end{array}$ & $\Delta^{\mathrm{a}}$ & $\begin{array}{r}\text { Eqn. } \\
\text { (2) }\end{array}$ & $\Delta^{\mathrm{a}}$ & $\begin{array}{r}\text { Eqn. } \\
\text { (3) }\end{array}$ & $\Delta^{\mathrm{a}}$ \\
\hline 1 & $\mathrm{Cl}$ & $\mathrm{OCH}_{2} \mathrm{CCH}$ & 7.60 & 7.30 & 0.30 & 7.56 & 0.04 & 7.36 & 0.24 \\
\hline 2 & $\mathrm{Cl}$ & $\mathrm{OCH}_{2} \mathrm{CHCH}_{2}$ & 7.50 & 7.20 & 0.30 & 7.32 & 0.18 & 7.20 & 0.30 \\
\hline 3 & $\mathrm{Cl}$ & $\mathrm{OCH}_{3}$ & 7.50 & 7.30 & 0.30 & 7.01 & 0.49 & 7.43 & 0.07 \\
\hline 4 & $\mathrm{Cl}$ & $\mathrm{OH}$ & 7.20 & 7.20 & 0.00 & 6.89 & 0.31 & 6.88 & 0.32 \\
\hline 5 & $\mathrm{Cl}$ & $\mathrm{CH}_{2} \mathrm{OCH}_{3}$ & 7.10 & 7.10 & 0.00 & 7.01 & 0.09 & 6.69 & 0.41 \\
\hline 6 & $\mathrm{Cl}$ & $\mathrm{NHSO}_{2} \mathrm{C}_{2} \mathrm{H}_{5}$ & 7.10 & 7.10 & 0.00 & 7.28 & -0.18 & 7.24 & -0.14 \\
\hline 7 & $\mathrm{Cl}$ & $\mathrm{OCOCH}_{3}$ & 7.10 & 7.20 & 0.00 & 7.10 & 0.00 & 7.47 & -0.37 \\
\hline 8 & $\mathrm{Cl}$ & $\mathrm{CH}_{3}$ & 7.00 & 7.10 & -0.10 & 6.77 & 0.23 & 6.93 & 0.07 \\
\hline 9 & $\mathrm{Cl}$ & $\mathrm{H}$ & 6.80 & 6.90 & -0.10 & 7.14 & -0.34 & 6.31 & 0.49 \\
\hline 10 & $\mathrm{Cl}$ & $\mathrm{NHSO}_{2} \mathrm{CH}_{3}$ & 6.70 & 6.90 & -0.20 & 6.90 & -0.20 & 7.37 & -0.67 \\
\hline 11 & $\mathrm{Cl}$ & $\mathrm{OC}_{6} \mathrm{H}_{5}$ & 6.60 & 6.50 & 0.10 & 6.78 & -0.18 & 6.65 & -0.05 \\
\hline 12 & $\mathrm{Cl}$ & $\mathrm{Cl}$ & 6.50 & 6.70 & -0.20 & 6.72 & -0.22 & 6.61 & -0.11 \\
\hline 13 & $\mathrm{Cl}$ & $\mathrm{Br}$ & 6.50 & 6.20 & 0.30 & 6.65 & -0.15 & 6.16 & 0.34 \\
\hline 14 & $\mathrm{Cl}$ & $\mathrm{C}_{6} \mathrm{H}_{5}$ & 6.30 & 6.30 & 0.00 & 6.29 & 0.01 & 6.44 & -0.14 \\
\hline 15 & $\mathrm{Cl}$ & $\mathrm{O}-\left(4-\mathrm{NHSO}_{2} \mathrm{Et}\right)$ phenyl & 6.60 & p & I & 6.46 & 0.14 & 6.53 & 0.07 \\
\hline 16 & $\mathrm{Cl}$ & O-(4-methoxy)phenyl & 6.70 & / & I & 6.94 & -0.24 & 6.92 & -0.22 \\
\hline 17 & $\mathrm{Cl}$ & O-(4-Cl)phenyl & 6.70 & I & I & 6.75 & -0.05 & 6.51 & 0.19 \\
\hline 18 & $\mathrm{Cl}$ & $\mathrm{O}-\left(4-\mathrm{NO}_{2}\right)$ phenyl & 6.80 & I & I & 6.73 & 0.07 & 6.39 & 0.41 \\
\hline 19 & $\mathrm{Cl}$ & $\mathrm{O}-\left(4-\mathrm{OCH}_{2} \mathrm{CO}_{2} \mathrm{Et}\right)$ phenyl & 9.00 & I & I & 7.44 & 1.56 & 6.71 & 2.29 \\
\hline 20 & O-(4-Cl)benzyl & $\mathrm{H}$ & 7.90 & l & I & I & I & 4.38 & 3.52 \\
\hline 21 & O-(4-Cl)benzyl & $\mathrm{NH}_{2}$ & 4.90 & I & I & I & I & 4.97 & -0.07 \\
\hline 22 & O-(4-Cl)benzyl & $\mathrm{Cl}^{2}$ & 5.00 & I & I & I & I & 5.30 & -0.30 \\
\hline 23 & $\mathrm{Br}$ & $\mathrm{H}$ & 6.10 & I & I & I & I & 6.61 & -0.51 \\
\hline 24 & $\mathrm{~F}$ & $\mathrm{H}$ & 5.30 & I & I & I & I & 5.50 & -0.20 \\
\hline 25 & $\mathrm{NO}_{2}$ & $\mathrm{H}$ & 5.20 & I & I & I & I & 5.48 & -0.28 \\
\hline 26 & $\mathrm{OCH}\left(\mathrm{CH}_{3}\right)_{2}$ & $\mathrm{H}$ & 5.10 & I & l & l & I & 4.97 & 0.13 \\
\hline
\end{tabular}

In Eqns. (1) and (2), the $B$ parameter is the 'minimum width' STERIMOL value for the minimum perpendicular distance from the $L$ axis to the tangential
Table 1. Structure and herbicidal activity of $\mathrm{N}$-phenyl triazolinone derivatives

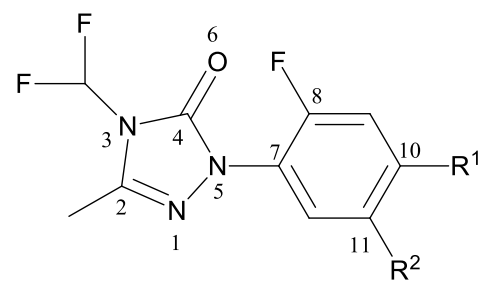

${ }^{\mathrm{a}} \Delta$ : Difference between observed (Obs) and calculated values; ${ }^{\mathrm{b}}$ not calculated. surface plane of the substituents; $L$ and $B_{5}$ are the revised STERIMOL parameters for substituents with fully extended and staggered conformations at the designated positions. The $L$ parameter is the length (A) along the bond axis that connects the $\alpha$ atom of the substituent with the rest of the molecule. The $B_{5}$ parameter is for the largest width $(\AA)$ perpendicular to the $L$ axis. The reference point of STERIMOL parameters is shifted to the values of hydrogen; this is represented by placing $\Delta$ in front of the notations. Although the correlation coefficient $\left(r^{2}\right)$ of Eqn. (2) was lower than that of Eqn. (1), the equation was highly significant (over 99.8\%). The relatively low $r^{2}$ value might be due to the narrow distribution of the $p \mathrm{I}_{50}$ values, which ranged from 6.5 to 7.6. Although compound 19 was not included, Eqn. (2) provided a good prediction of compound 17 activity.

In fact, Theodoridis et al..$^{[36]}$ provided the herbicidal activity of all 26 compounds shown in Table 1, but Eqns. (1) and (2) only included 14 and 18 compounds, respectively. In order to establish QSAR equations with higher predictability, the equilibrium geometries, electronic structures, and electrostatic potentials of all 26 compounds were investigated with the density functional theory (DFT) method. [37] Quantum chemical descriptors, including electrophilic or nucleophilic frontier electron densities and net atomic charges, were derived from the DFT calculations. Based on these quantum chemical descriptors, a DFT-QSAR Eqn. (3) was derived by Zhang et al. ${ }^{[37]}$

In Eqn. (3), $Q_{C_{1}}$ represents the net atomic charges of the carbon atom at position $11 ; f_{N_{s}}^{E}$ and $f_{C}^{N}$ are the electrophilic and nucleophilic frontier electron densities, respectively, of the 5-position nitrogen atom and the 10position carbon atom, respectively. Interestingly, replacing the DFT-based parameters with quantum chemical descriptors derived from semiempirical 


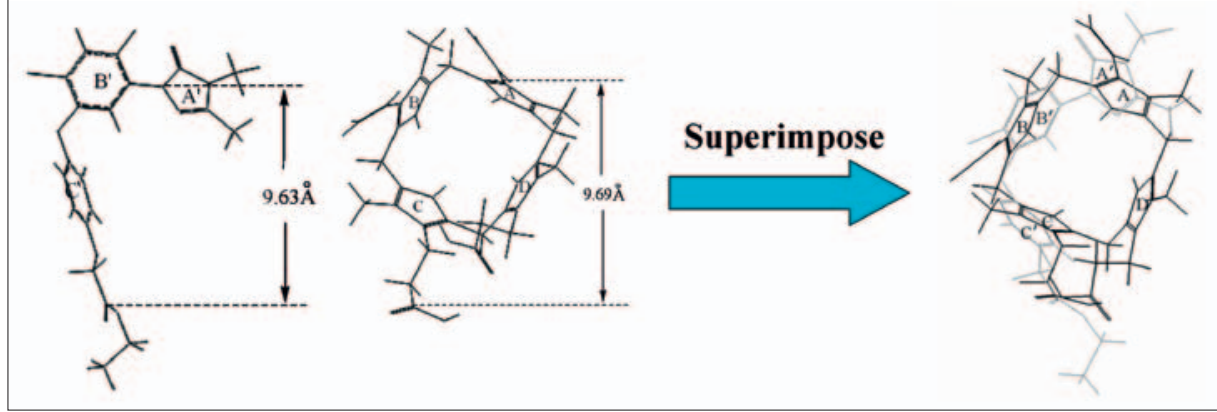

Fig. 5. Comparison of the molecular shapes of compound 19 (left) and protoporphyrinogen IX (middle); superimposition (right) shows compound 19 in light grey.

methods (e.g. PM3) resulted in poor predictions. It should be noted that compound 19 was not included in any of the above Eqns. (1), (2), or (3). Nevertheless, the DFT calculations did provide a rational explanation for the very potent activity of this compound. By superimposing compound 19 and the substrate (Fig. $5)$, it was clear that their conformations were very similar. The distance between the nitrogen (or carbon) atom at the 5-position and the carbonyl carbon atom was $9.63 \AA$ for compound $\mathbf{1 9}$ and 9.69 $\AA$ for the substrate. Further molecular dynamic simulation results suggested that compound 19 was highly similar to rings $\mathrm{A}, \mathrm{B}$, and $\mathrm{C}$ of the substrate. Furthermore, the side-chain carbonyl oxygen atom of compound 19 formed two hydrogen bonds with Thr68 in the mtPPO binding pocket (unpublished results).

One of the most important types of PPO inhibitors is the cyclic imide. For years, many cyclic imide compounds, including flumioxazin, flumicloracpentyl, cinidonethyl, and profluazol, have been developed as commercial herbicides. In 1980, Fujita et al. were the first to report a QSAR analysis of herbicidal $\mathrm{N}$-aryl-3,4,5,6-tetrahydro-phthalimides and related cyclic imides, like 3-aryl1,5- tetramethylenehydantoins, 4-aryl-1,2tetramethylene-triazolidine-3,5-diones, and their corresponding thiocarbonyl compounds. ${ }^{[38]}$ They found that the substituents, in terms of the STERIMOL parameters ( $\Delta L$ and $\Delta B_{5}$ ), had significant steric effects, particularly at ortho and para positions. This was confirmed in 1988 by Wakabayashi et al., who carried out a QSAR analysis for forty $N$-aryl3,4,5,6-tetrahydro-phthalimides. [39] However, Nicolaus et al.[40] performed a subsequent QSAR analysis for $\mathrm{N}$ aryl-3,4,5,6-tetrahydro-phthalimides and showed that only the hydrophobicity of the substituents significantly affected the herbicidal activity of those compounds. These earlier QSAR studies were limited to the use of physicochemical descriptors or semiempirical electronic structure descriptors; these descriptors were established to study the effects of substituents in the $N$-phenyl moiety, but the imine moiety was maintained invariant. Therefore, Wan et al. ${ }^{[41]}$ established the first DFT-QSAR equation for 26 cyclic imide compounds with diverse imine moieties and substituents in the $N$-phenyl moiety (Fig. 6). Eqn. (4) was derived based on three quantum chemical descriptors at the level of B3LYP/6-31G(d,p); $F_{C^{*}}^{N}$, the weighted nucleophilic frontier electron densities of the carbon atom with the asterisk (see Fig. 6); $Q_{N^{* *}}$ the net atomic charge of the nitrogen atom with the double asterisk, and $\alpha$, the molecular polarizability. The leaveone-out cross-validation analysis indicated that Eqn. (4) had very good predictability, descriptor, which described the steric effects of a substituent by characterizing its electron cloud volume, based on the DFT calculation; with this, they established a QSAR equation with very good predictive ability for 35 sulfonylurea compounds. It should be noted that, although a large number of quantum-chemical descriptors had been defined and applied successfully in QSAR studies, no quantum-chemical descriptor for steric effects had been previously developed.

The above examples showed that the DFT-based quantum chemical descriptors could provide more predictive equations than the corresponding semiempirical quantum chemical descriptors and traditional physiochemical parameters. Another advantage of the DFT-QSAR approach is that it can determine the bioactive conformations of small molecules. Many techniques, including $\mathrm{X}$-ray crystallography diffraction, nuclear magnetic resonance, and molecular dynamic simulation, have been developed and used for the analyses of bioactive conformations of small molecules. However, these techniques always have drawbacks, like the dependence on expensive instruments, difficulty in obtaining the target enzyme, and large time requirements. Importantly, most

$$
\begin{aligned}
& p \mathrm{I}_{50}=-5.7414( \pm 0.0961)+0.1424( \pm 0.0192) \alpha-0.0003( \pm 0.0000) \alpha^{2} \\
& -0.4546( \pm 0.0542) F_{C^{*}}^{N}+0.2974( \pm 0.1932) Q_{N^{* *}} \\
& \mathrm{n}=26, r^{2}=0.87, s=0.4899, F=35.98
\end{aligned}
$$

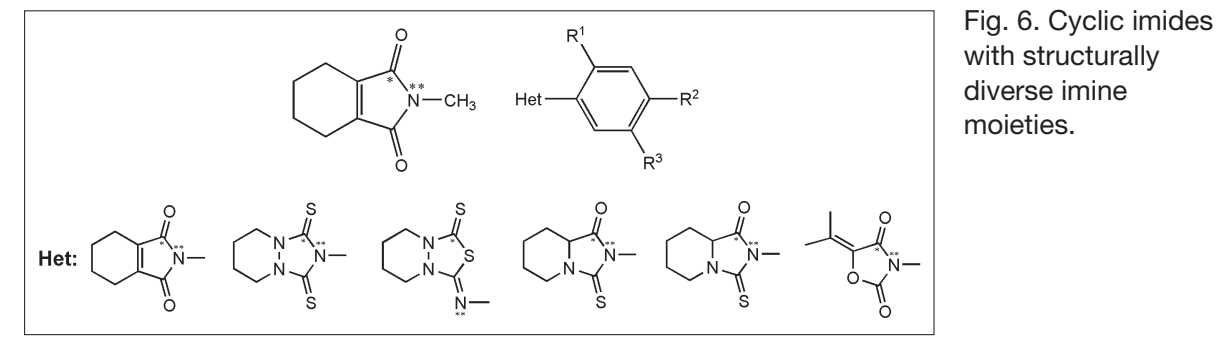

due to its high cross-validated coefficient of 0.87 .

In fact, the electronic structure theory has become an effective, powerful tool for predicting a wide range of molecular properties, including geometries, energetics, reactivity, and spectroscopic properties. DFT is a powerful computational approach with relatively low computational cost and reasonable accuracy; it has been widely applied to investigate the precise electronic characteristics of molecular structures, a key factor in interactions between receptors and the ligands. For example, $\mathrm{Xi}$ et $a l .{ }^{[42]}$ defined the first quantum-chemical active sites for pesticides are unknown. Thus, it is nearly impossible to determine the bioactive conformation of a pesticide molecule. QSAR analysis has become one of the most effective approaches for optimizing lead compounds and designing new drugs; however, it had not previously been used to analyze bioactive conformations.

Recently, Zhang et al. ${ }^{[43]}$ extended the DFT-QSAR strategy to predictions of bioactive conformations. They focused on a series of cyclic imide derivatives (Fig. 7). First, the DFT calculation was used to optimize the geometries of 


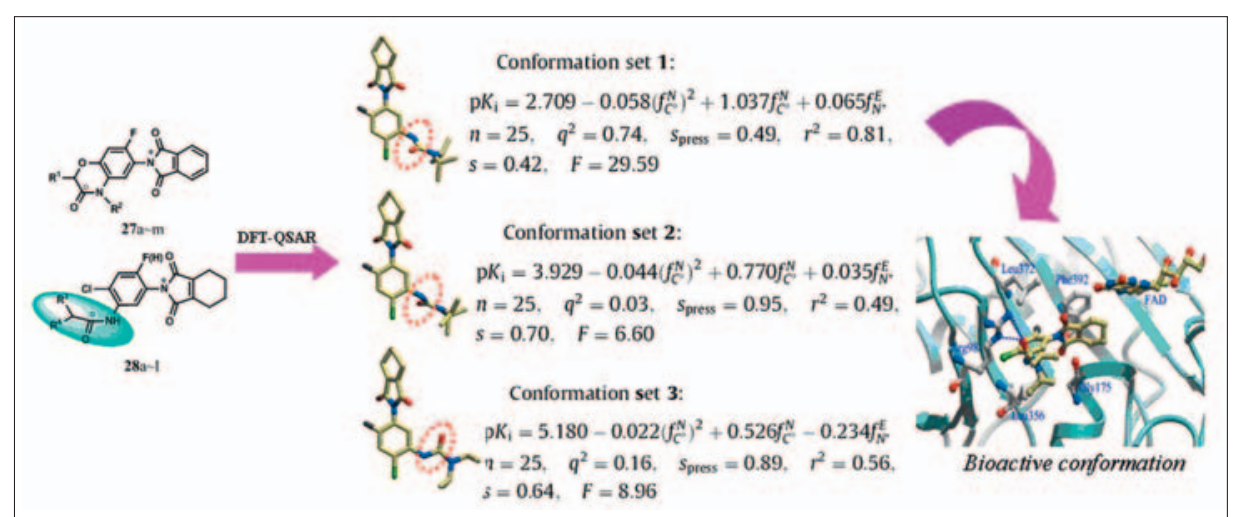

Fig. 7. Bioactive conformation of cyclic imines was derived by combining the density functional theory (DFT) and quantitative structure-activity relationship (QSAR) analyses.

compounds 27 and 28. Only one kind of equilibrium conformation was obtained for compounds $\mathbf{2 7} \mathbf{a}-\mathbf{m}$, due to their rigid structures; in contrast, three different equilibrium conformations were obtained for compounds $\mathbf{2 8 a}-\mathbf{l}$, due to their flexible side chains. Then, three QSAR equations were established for the three different equilibrium conformations with DFT-based descriptors. The predicted bioactive conformation was selected as the equilibrium conformation with the highest cross-validation correlation coefficient $\left(q^{2}\right)$ and traditional correlation coefficient $\left(r^{2}\right)$. Finally, the 'real' bioactive conformation was derived by performing further potential energy surface scans, molecular docking tests, and molecular dynamic simulations. Interestingly, the DFT-QSAR-derived conformation was very close to the 'real' bioactive conformation. Thus, the DFTQSAR approach could be used as a simple alternative to bioactive conformation analysis of small molecules, particularly when the three-dimensional structure of a protein is unknown. The combination of DFT calculations with QSAR analysis has extended the application of the classical QSAR method.

\section{New PPO Inhibitors}

The characteristics exhibited by PPOinhibiting herbicides have attracted the attention of pesticide chemists worldwide. Great effort has focused on the synthesis of new PPO inhibitors, and some promising compounds with excellent herbicidal activity have been discovered over the last decade.

According to the substituents of the pyrrole ring, the protoporphyrinogen-IX molecule can be divided into two distinct regions. One half of the molecule has a predominantly lipophilic profile, and the other half, with the propionic acid chains, has a more hydrophilic profile. Although herbicides were thought to compete with protoporphyrinogen-IX, in fact, most inhibi- tors mimicked two of the pyrrole rings in protoporphyrinogen-IX. In 1995, the first inhibitor (29; Scheme 2) was designed that mimicked the three rings of the propionate portion of protoporphyrinogen-IX. ${ }^{[44]}$ In addition to the triazolinone, a number of other heterocyclic rings were investigated. Because phenyluracils had been known to have herbicidal activity since the late 1980 s, one group replaced the triazolinone ring with dihydropyrimidine-2,4dione. This resulted a new class of herbicidal molecules. ${ }^{[18]}$ In addition, placing a trifluoromethyl group at position 6 on the dihydropyrimidine-2,4-dione ring greatly increased herbicidal activity. Further structural optimization eventually produced four important commercial herbicides, including flupropacil, ${ }^{[45]}$ benzfendizone, ${ }^{[18]}$ butafenacil, ${ }^{[46]}$ and saflufenacil ${ }^{[47]}$ (Fig. 3 ). Among these dihydropyrimidine-2,4dione herbicides, saflufenacil was recently registered as a selective herbicide for the control of broadleaf weeds in pre-emergence or post-emergence applications for several crops, including cotton, soybeans, and sunflowers. It can also be used in fallow croplands and non-agricultural areas. Saflufenacil is highly active, and can be
$\mathrm{Cl}$ at $\mathrm{C}(2)$ and $\mathrm{Cl}$ at $\mathrm{C}(4)$; in addition, the groups at the $\mathrm{C}(5)$ position had important effects on both the activity and crop selectivity. Therefore, structural optimizations have always focused on the substituents at the $\mathrm{C}(5)$ position. The $\mathrm{C}(5)$ position is ortho to the $\mathrm{C}(4)$ position; therefore, an interesting, effective strategy emerged when a fused benzoheterocyclic ring was placed at the $\mathrm{C}(4)$ and $\mathrm{C}(5)$ positions. In addition, some PPO inhibitors have been synthesized with a fused $N$-phenyl heterocyclic moiety placed at $\mathrm{C}(5)$ and $\mathrm{C}(6)$. Fig. 8 shows a number of compounds with five- or six-membered, fused benzoheterocyclic rings, ${ }^{[48-57]}$ including dihydrobenzooxazin-2-one, dihydrobenzooxazin-3-one, benzoisoxazolinone, quinolin-2-one, benzoisoxazol-3-one, benzoimidazole, benzooxazole, benzothiazole, etc. Although many benzoheterocyclic compounds with promising herbicidal activity have been synthesized, only one dihydrobenzooxazin-2-one compound, flumioxazin, was commercialized. [58] Recently, Jiang et al. ${ }^{[57,59]}$ designed and synthesized a series of benzothiazole derivatives as $\mathrm{PPO}$ inhibitors; some of these displayed good herbicidal activity and crop selectivity. For example, compound $\mathbf{3 0}$ showed good PPO inhibition ( $\left.k_{i}=1.42 \mathrm{nM}, \mathrm{hPPO}\right)$ and a broad spectrum post-emergence herbicidal activity at a concentration of $37.5 \mathrm{~g}$.ai/ha (Fig. 9). Compounds 31, 32, 33, and 34 exhibited broad spectrum herbicidal activity comparable to sulfentrazone at a concentration of 37.5 g.ai/ha. Interestingly, compounds $\mathbf{3 1}$ and $\mathbf{3 2}$ were highly safe on wheat and maize, even above 300 g.ai/ha. However, these crops are very sensitive to sulfentrazone, even at $150 \mathrm{~g}$.ai/ha. These results indicated that compounds $\mathbf{3 1}$ and 32 showed potential as new herbicides for weed control in wheat or maize fields.

As mentioned previously, the DFTQSAR results for the series of cyclic

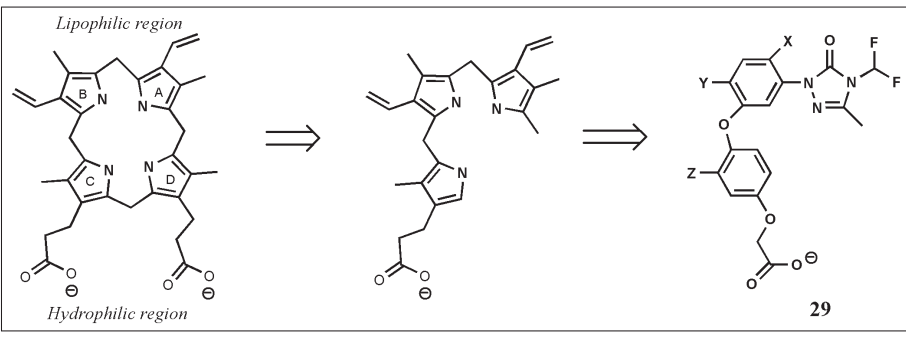

Scheme 2. An inhibitor (29) designed to mimic three rings of protoporphyrinogen IX (left).

used to control weeds that have evolved resistance to AHAS-inhibiting herbicides and glyphosate.

From the perspective of chemical structure, most $N$-phenyl heterocyclic PPO inhibitors are 1,2,4,5-tetrasubstituted benzene derivatives. Of the phenyl substitution patterns investigated, those that led to the most active compounds had an F or imides indicated that ${ }^{[41]}$ the approximate nucleophilic, superdelocalizability of the carbon atom on one of the carbonyl groups played an important role in determining the activity of PPO inhibitors. Increasing the ability of the carbonyl group to accept electrons from a receptor causes an increase in the PPO inhibitory activity. Furthermore, only one of the carbonyl 


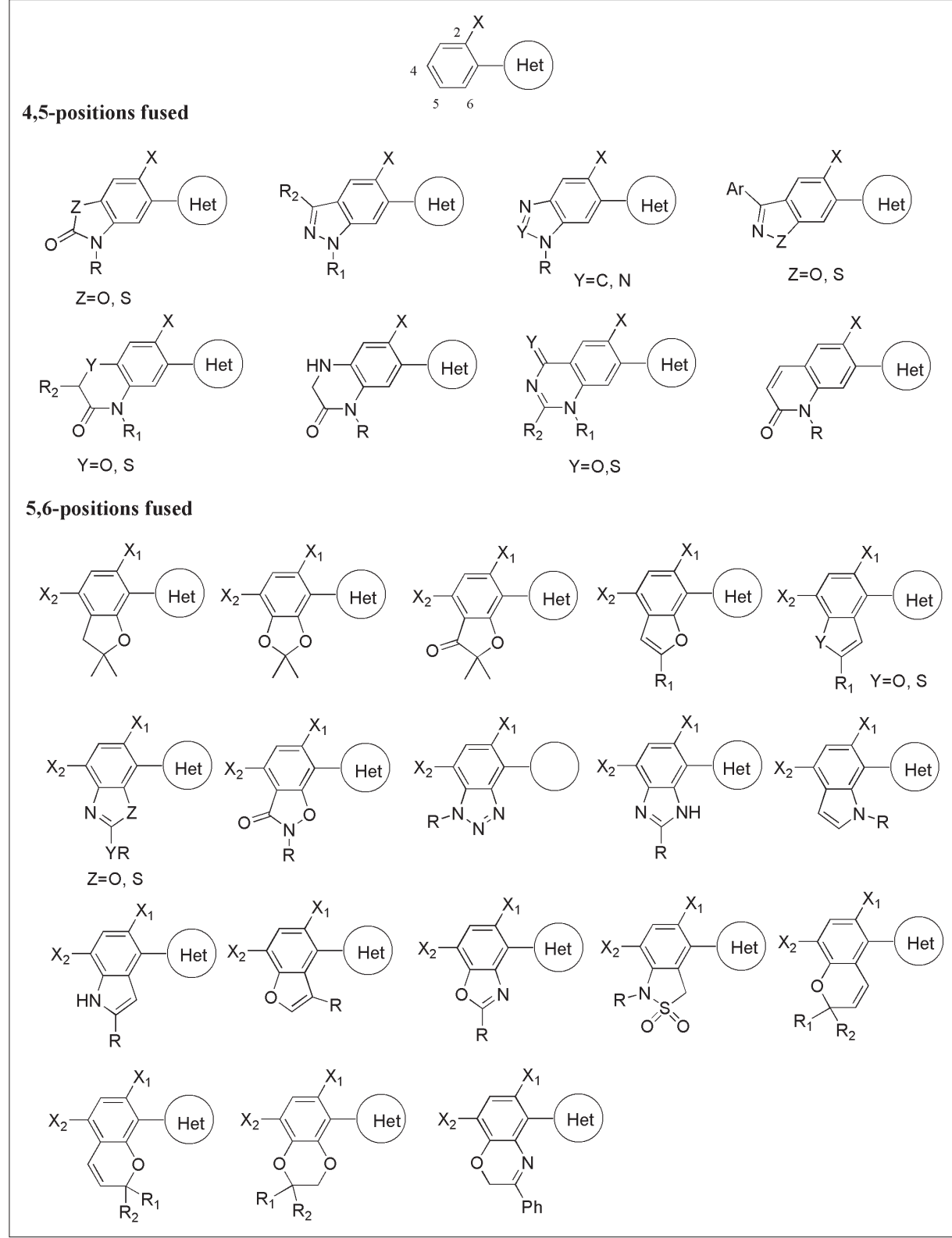

Fig. 8. Protoporphyrinogen oxidase inhibitors with benzoheterocyclic $N$-phenyl moieties.

groups was essential for inhibitor activity. Prompted by these DFT-QSAR results, Zhang et al. ${ }^{[60]}$ designed and synthesized a series of $N$-phenyl-1H-pyrrol-2-ones as PPO inhibitors. Compounds $\mathbf{3 3}$ and $\mathbf{3 4}$ were highly potent hPPO inhibitors, with $k_{\text {. }}$ values of $95 \mathrm{nM}$ and $120 \mathrm{nM}$, respectively. Both compounds also exhibited broad spectrum herbicidal activity at a concentration of 150 g.ai/ha.

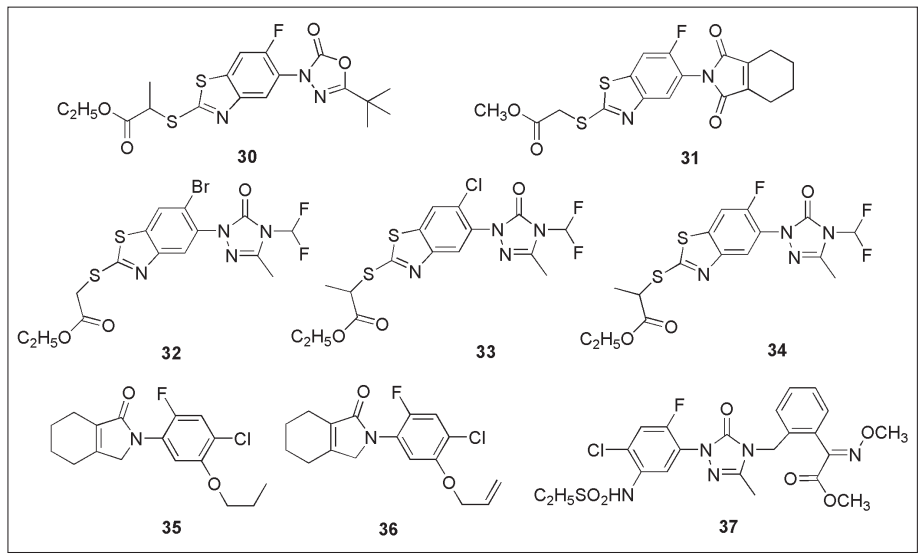

Fig. 9. Some new protoporphyrinogen oxidase (PPO) inhibitors with promising herbicidal activity.

Received: October 16, 2011

[1] R. Poulson, W. J. Polglase, J. Biol. Chem. 1975 , 250, 1269.

[2] S. Arnould, M. Takahashi, J. M. Camadro, Proc. Natl. Acad. Sci. U. S. A. 1999, 96, 14825.

[3] G. F. Hao, Y. Tan, N. X. Yu, G. F. Yang, $J$. Comput. Aided Mol. Des. 2011, 25, 213.

[4] I. Lermontova, E. Kruse, H. P. Mock, B. Grimm, Proc. Natl. Acad. Sci. U. S. A. 1997, 94, 8895.

[5] D. A. Brenner, J. R. Bloomer, N. Engl. J. Med. 1980, 302,765 .

[6] J. C. Deybach, H. de Verneuil, Y. Nordmann, Hum. Genet. 1981, 58, 425. 
[7] P. N. Meissner, R. S. Day, M. R. Moore, P. B. Disler, E. Harley, Eur. J. Clin. Invest. 1986, 16 , 257.

[8] S. D. Whatley, H. Puy, R. R. Morgan, A. M. Robreau, A. G. Roberts, Y. Nordmann, G. H. Elder, J. C. Deybach, Am. J. Hum. Genet. 1999 65, 984.

[9] Y. Nordmann, H. Puy, Clin. Chim. Acta 2002, 325, 17.

[10] R. S. Day, Semin. Dermatol. 1986, 5, 138.

[11] M. H. Maneli, A. V. Corrigall, H. H. Klump, L. M. Davids, R. E. Kirsch, P. N. Meissner, Biochim. Biophys. Acta 2003, 1650, 10.

[12] V. H. Fingar, T. J. Wieman, K. S. McMahon, P. S. Haydon, B. P. Halling, D. A. Yuhas, J. W. Winkelman, Cancer Res. 1997, 57, 4551.

[13] B. P. Halling, D. A. Yuhas, V. F. Fingar, J. W. Winkelmann, 'Protophyrinogen oxidase inhibitors for tumor therapy, in Porphyric pesticides: Chemistry, toxicology and pharmaceutical applications', American Chemical Society, Washington, DC, 1994.

[14] M. Koch, C. Breithaupt, R. Kiefersauer, J Freigang, R. Huber, A. Messerschmidt, EMBO J. 2004, 23, 1720.

[15] T. K. Gitsopoulos, R. J. Froud-Williams, Weed Res. 2004, 44, 329.

[16] G. Theodoridis, Pestic. Sci. 1997, 50, 283.

[17] M. Hiraki, S. Ohki, Y. Sato, I. Jablonkai, P Böger, K. Wakabayashi, Pestic. Biochem. Physiol. 2001, 70, 159.

[18] G. Theodoridis, J. T. Bahr, F. W. Hotzman, S. Saroj, D. P. Suarez, Crop Prot. 2000, 19, 533.

[19] K. Hirai, T. Futikami, A. Murata, H. Hirose, US Patent 4818272, 1989

[20] J. W. Lyga, R. M. Patera, G. Theodoridis, B. P. Halling, F. W. Hotzman, M. J. Plummer, J. Agric. Food Chem. 1991, 39, 1667

[21] K. A. Simmons, J. A. Dixson, B. P. Halling, E L. Plummer, M. J. Plummer, J. M. Tymonko, R. J. Schmidt, M. J. Wyle, C. A. Webster, et al., J. Agric. Food Chem. 1992, 40, 297.

[22] F. D. Hess, Weed Sci. 2000, 48, 160.

[23] H. R. Corradi, A. V. Corrigall, E. Boix, C. G. Mohan, E. D. Sturrock, P. N. Meissner, K. R Acharya, J. Biol. Chem. 2006, 281, 38625.
[24] X. Qin, L. Sun, X. Wen, X. Yang, Y. Tan, H. Jin, Q. Cao, W. Zhou, Z. Xi, Y. Shen, J. Struct. Biol. 2010, $170,76$.

[25] X. H. Qin, Y. Tan, L. L. Wang, Z. F. Wang, B. F. Wang, X. Wen, G. F. Yang, Z. Xi, Y. Q. Shen, FASEB J. 2011, 25, 653.

[26] http://www.weedscience.org/summary/HRA Cchem.asp.

[27] http://www.weedscience.org/Summary/Uspecies MOAasp? lstMOAID $=8 \&$ FmHRACGroup $=G o$.

[28] M. D. Owen, I. A. Zelaya, Pest Manag. Sci. 2005, 61, 301 .

[29] W. L. Patzoldt, A. G. Hager, J. S. McCormick, P. J. Tranel, Proc. Natl. Acad. Sci. U. S. A. 2006, 103, 12329.

[30] G. F. Hao, X. L. Zhu, F. Q. Ji, L. Zhang, G. F. Yang, C. G. Zhan, J. Phys. Chem. B 2009, 113, 4865.

[31] X. Li, D. Nicholl, Pest Manag. Sci. 2005, 61, 277.

[32] R. M. S. Mulwa, L. M. Mwanza, African J. Biotech. 2006, 5, 396

[33] D. Werck-Reichhart, A. Hehn, L. Didierjean, Trends Plant Sci. 2000, 5, 116.

[34] H. Nakajima, A. Nagasawa, US Patent 6570070 , 2003

[35] T. Fujita, A. Nakayama in 'Structureactivity relationship and molecular design of peroxidizing herbicides with cyclic imide structures and their relatives', Eds. P. Böger, K. Wakabayashi, Springer-Verlag Berlin Heidelberg, 1999.

[36] G. Theodoridis, Pestic. Sci. 1997, 50, 283.

[37] L. Zhang, J. Wan, G. Yang, Bioorg. Med. Chem. 2004, 12, 6183

[38] H. Ohta, T. Jikihara, K. Wakabayashi, T. Fujita, Pestic. Biochem. Physiol. 1980, 14, 153.

[39] K. Wakabayashi, J. Pestic. Sci. 1988, 13, 337. [40] B. Nicolaus, G. Sandmann, P. Böger, Z. Naturforsch C. 1993, 48, 326.

[41] J. Wan, L. Zhang, G. Yang, C. G. Zhan, J. Chem. Inf. Comput. Sci. 2004, 44, 2099.

[42] Z. Xi, Z. Yu, C. Niu, S. Ban, G. Yang, J. Comput. Chem. 2006, 27, 1571.

[43] L. Zhang, G. F. Hao, Y. Tan, Z. Xi, M. Z. Huang, G. F. Yang, Bioorg. Med. Chem. 2009, 17, 4935.
[44] G. Theodoridis, M. Poss Kathleen, W. Hotzman Frederick in 'Herbicidal 1-(2,4-Dihalo5-phenoxyphenyl)-4-difluoromethyl-4,5dihydro-3-methyl-1,2,4-triazolin-5(1H)-one Derivatives', Eds. D. R. Baker, J. G. Fenyes, G. S. Basarak, American Chemical Society, 1995, pp. 78.

[45] Y. Sanemitsu, S. Kawamura, J. Pestic. Sci. 2008, 33, 175 .

[46] F. E. Dayan, S. O. Duke, K. Grossmann, Weed Sci. 2010, 58, 340

[47] K. Grossmann, J. Hutzler, G. Caspar, J Kwiatkowski, C. L. Brommer, Weed Sci. 2011, 59, 290.

[48] W. Hong, M. Schafer, T. M. Stevenson, WO Patent 9506643, 1995.

[49] L. L. Maravetz, G. Theodoridis, WO Patent 9712884, 1997.

[50] S. D. Crawford, L. L. Maravetz, G. Theodoridis, US Patent 5661108, 1997.

[51] V. Kameswaran, WO Patet 0049004, 2000.

[52] J. Satow, Y. Kudo, WO Patent 9814452, 1998.

[53] M. W. Drewes, R. Andree, M. Dollinger, DE Patent 19530451, 1997.

[54] G. Theodoridis, US Patent 5521147, 1996.

[55] R. Reinhard, G. Hamprecht, P. Schafer, WO Patent 2000028822, 2000.

[56] B. Kamireddy, W. M. Murray, WO Patent 9533746, 1995.

[57] L. L. Jiang, Y. Tan, X. L. Zhu, Z. F. Wang, Y. Zuo, Q. Chen, Z. Xi, G. F. Yang, J. Agric. Food Chem. 2010, 58, 2643.

[58] M. Z. Huang, F. X. Luo, H. B. Mo, Y. G. Ren, X. G. Wang, X. M. Ou, M. X. Lei, A. P. Liu, L. Huang, M. C. Xu, J. Agric. Food Chem. 2009 57, 9585 .

[59] L. L. Jiang, Y. Zuo, Z. F. Wang, Y. Tan, Q. Y. Wu, Z. Xi, G. F. Yang, J. Agric. Food Chem 2011, 59, 6172 .

[60] L. Zhang, Y. Tan, N. X. Wang, Q. Y. Wu, Z. Xi, G. F. Yang, Bioorg. Med. Chem. 2010, 18, 7948

[61] Y. P. Luo, L. L. Jiang, G. D. Wang, Q. Chen, G. F. Yang, J. Agric. Food Chem. 2008, 56, 2118. 